\title{
Spontaneous Generation of Torsion Coupling of Electroweak Massive Gauge Bosons
}

\author{
Hagen Kleinert* \\ Institut für Theoretische Physik, \\ Freie Universität Berlin, Arnimallee 14, 14195 Berlin, Germany
}

\begin{abstract}
We derive the coupling to torsion of massive electroweak vector bosons generated by the Higgs mechanism.
\end{abstract}

1. In the Poincaré gauge formulation [1] of Einstein-Cartan gravity, the electromagnetic field cannot couple minimally to torsion since this would destroy gauge invariance. If torsion does not propagate, so that the torsion field is confined to exist only inside of elementary particles, this would not matter, since the propagation of photons within matter is much stronger modified by electromagnetic dispersion and absorption than by any conceivable gravitational torsion field. In the physically only interesting case of a propagating torsion, however, a non-gauge-invariant coupling would have the fatal consequence that the photon would become massive. Since the photon mass can be estimated experimentally to be smaller than $3 \times 10^{-27} \mathrm{eV}$, this would lead to the conclusion that the torsion field in the universe is so small that there is no need for contriving theories for its possible properties, or that the photon does not couple to torsion via the affine covariant derivative.

Massive vector bosons, on the other hand, such as the $\rho$-meson, whose wave function has a large amplitude in a state of a quark and an antiquark in an $s$-wave spin triplet channel, should certainly couple to torsion via their quark content.

By analogy with photons, the fundamental action describing electroweak processes should contain no minimally coupled torsion in the gradient terms of the bare vector bosons $W$ and $Z$. However, these particles acquire a mass via the Meissner-Higgs effect which makes them essentially composite particles, their fields being a mixture of the original massless vector fields and the Higgs fields. By analogy with the massive $\rho$-vector field, we could expect that also the massive electroweak vector fields couple to torsion, and the question arises how the Meissner-Higgs effect is capable of generating such a coupling.

In general, we do not know the answer to this problem. In this note we would like to show how such a coupling does arise if the torsion is of the gradient type

$$
S_{\mu \nu}^{\lambda}(x)=\frac{1}{2}\left[\delta_{\mu}^{\lambda} \partial_{\nu} \sigma(x)-\delta_{\nu}{ }^{\lambda} \partial_{\mu} \sigma(x)\right] .
$$

2. In the standard Poincaré gauge formulation [1] of gravity it is immediately obvious that a minimally-coupled scalar Higgs field with an action

$$
\mathcal{A}[\phi]=\int d^{4} x \sqrt{-g}\left(\frac{1}{2} g^{\mu \nu}\left|\nabla_{\mu} \phi \nabla_{\nu} \phi\right|-\frac{m^{2}}{2}|\phi|^{2}-\frac{\lambda}{4}\left|\phi^{2}\right|^{2}\right)
$$

cannot equip a previously uncoupled massless vector field with a torsion coupling. For simplicity, we consider only a simple Ginzburg-Landau-type theory with a complex field to avoid inessential complications. As usual, $g=\operatorname{det} g_{\mu \nu}$ denotes the determinant of the metric $g_{\mu \nu}(x)$, and $\nabla_{\mu}$ is the electromagnetic covariant derivatives $\nabla_{\mu}=\partial_{\mu}-i e V_{\mu}$. The square mass is negative, so that the Higgs field has a nonzero expectation value with $|\phi|^{2}=-m^{2} / \lambda$. From the derivative term, the vector field acquires a mass term $e^{2}|\phi|^{2} V^{\mu} V_{\mu} / 2$, leading to the a free part of the vector boson action

$$
\mathcal{A}[V]=\int d^{4} x \sqrt{-g}\left(-\frac{1}{4} F_{\mu \nu} F^{\mu \nu}-\frac{e^{2} m^{2}}{2 \lambda} V_{\nu} V^{\mu}\right),
$$

where $F_{\mu \nu}$ the covariant curl $F_{\mu \nu} \equiv \partial_{\mu} V_{\nu}-\partial_{\nu} V_{\mu}$. Of course, the covariant curl of the nonabelian electroweak vector bosons would also have self-ineractions, which can however be ignored in the present dicussion since we are only interested in the free-particle propagation.

Since the Meissner-Higgs effect creates the mass of the vector bosons by mixing the uncoupled bare vector boson with the scalar Higgs field, it is obvious that the massive vector bosons can couple to torsion only if the scalar Higgs

*Email: kleinert@physik.fu-berlin.de URL: http://www.physik.fu-berlin.de/ ${ }^{*}$ kleinert 
field has such a coupling. Indeed, it has recently been emphasized [2, 3] that, contrary to common belief [4], trajectories of scalar particles should be experience a torsion force. This conclusion was reached by a careful reinvestigation of the geometric properties of the variational procedure of the action. Taking into account the fact that in the presence of torsion parallelograms exhibit a closure failure, the variational procedure required a modification of this procedure [2,5.6] which led to the conclusion that scalar particles should move along autoparallel trajectories rather than geodesic ones as derived from a minimally coupled scalar field action [4]. The modification of the variational procedure was suggested to us by the close analogy of spaces with torsion with crystals containing defects $[7$.

3. So far, the classical trajectories have been quantized consistently with unitarity of time evolution only for a gradient torsion (11), and for a completely antisymmetric torsion [2]. In the case of gradient torsion the Schrödinger equation turns out to be driven by the Laplace operator $g^{\mu \nu} D_{\mu} D_{\nu}$, where $D_{\mu}$ is the covariant derivative involving the full affine connection $\Gamma_{\mu \nu}{ }^{\lambda}$, including torsion. It differs from the Laplace-Beltrami operator in torsion-free spaces $\Delta \equiv \sqrt{|g|}^{-1} \partial_{\mu} \sqrt{|g|} g^{\mu \nu} \partial_{\nu}$ by a term $-2 S^{\nu \lambda} \partial_{\nu}=-3\left(\partial^{\nu} \sigma\right) \partial_{\nu}$. This operator, however, is hermitian only in a scalar product which contains a factor $e^{-3 \sigma}[8]$. In the case of totally antisymmetric torsion, the two Laplace operators are equal and the original scalar product ensures hermiticity and thus unitarity of time evolution. Such a torsion drops also out from the classical equation of motion, so that autoparallel and geodesic trajectories coincide. For this reason we shall continue the discussion only for gradient torsion.

The gradient torsion has the advantage that it can be incorporated into the classical action of a scalar point particle in such a way that the modification of the variational procedure found in [5.6] becomes superfluous. The modified action reads for a massive particle [9]

$$
\mathcal{A}[x]=-m c \int d \tau e^{\sigma(x)} \sqrt{\left.g_{\mu \nu}(x)\right) \dot{x}^{\mu} \dot{x}^{\nu}}=-m c \int d s e^{\sigma(x(s))},
$$

where $\tau$ is an arbitrary parameter and $s$ the proper time. From the Euler-Lagrange equation we find that for $\tau=s$, the Lagrangian under the integral is a constant of motion, whose value is, moreover, fixed by the mass shell constraint

$$
L=e^{\sigma(x)} \sqrt{\left.g_{\mu \nu}(x)\right) \dot{x}^{\mu} \dot{x}^{\nu}} \equiv 1, \quad \tau=s .
$$

The necessity of a factor $e^{-3 \sigma(x)}$ in the scalar product discovered in [2] became the basis of a series of studies in general relativity [10,11]. In the latter work, the action of a relativistic free scalar field $\phi$ was found to be

$$
\mathcal{A}[\phi]=\int d^{4} x \sqrt{-g} e^{-3 \sigma}\left(\frac{1}{2} g^{\mu \nu}\left|\nabla_{\mu} \phi \nabla_{\nu} \phi\right|-\frac{m^{2}}{2}|\phi|^{2} e^{-2 \sigma}\right) .
$$

The associated Euler-Lagrange equation is

$$
D_{\mu} D^{\mu} \phi+m^{2} e^{-2 \sigma(x)} \phi=0
$$

whose eikonal approximation $\phi(x) \approx e^{i \mathcal{E}(x)}$ yields the following equation for the phase $\mathcal{E}(x)$ [1]:

$$
e^{2 \sigma(x)} g^{\mu \nu}(x)\left[\partial_{\mu} \mathcal{E}(x)\right]\left[\partial_{\nu} \mathcal{E}(x)\right]=m^{2} .
$$

Since $\partial_{\mu} \mathcal{E}$ is the momentum of the particle, the replacement $\partial_{\mu} \mathcal{E} \rightarrow m \dot{x}_{\mu}$ shows that the eikonal equation (\&) guarantees the constancy of the Lagrangian (5), thus describing autoparallel trajectories.

4. Apart from the factor $e^{-3 \sigma(x)}$ accompanying the volume integral, the $\sigma$-field couples to the scalar field like a dilaton, the power of $e^{-\sigma}$ being determined by the dimension of the associated term. If we therefore add to the free-field action (6) a quartic self-interaction to have a Meissner-Higgs effect, this self-interaction will not carry an extrafactor $e^{-\sigma}$, so that the proper Higgs action in the presence of gradient torsion reads

$$
\mathcal{A}[\phi]=\int d^{4} x \sqrt{-g} e^{-3 \sigma}\left(\frac{1}{2} g^{\mu \nu}\left|\nabla_{\mu} \phi \nabla_{\nu} \phi\right|-\frac{m^{2}}{2}|\phi|^{2} e^{-2 \sigma}-\frac{\lambda}{4}\left|\phi^{2}\right|^{2}\right)
$$

If $m^{2}$ is negative, and the torsion depends only weakly on spacetime, the Higgs field has a smooth vacuum expectation value

$$
|\phi|^{2}=-\frac{m^{2}}{\lambda} e^{-2 \sigma}
$$


The smoothness of the torsion field is required over a length scale of the Compton wavelength of the Higgs particle, i.e. over a distance of the order $1 / 20 \mathrm{GeV} \approx 10^{-15} \mathrm{~cm}$. For a torsion field of gravitational origin, this smoothness will certainly be guaranteed. From the gradient term in (9) we then extract in the gauge $\phi=$ real the mass term of the vector bosons

$$
\int d^{4} x \sqrt{-g} e^{-3 \sigma} \frac{1}{2} m_{V}^{2} e^{-2 \sigma(x)} V^{\mu} V_{\mu}
$$

where

$$
m_{V}^{2}=-\frac{e^{2}}{\lambda} m^{2}, \quad m^{2}<0
$$

Taking the physical scalar product in the presence of torsion into account, we obtain for the massive vector bosons the free-field action

$$
\mathcal{A}[V]=\int d^{4} x \sqrt{-g} e^{-3 \sigma}\left(-\frac{1}{4} F_{\mu \nu} F^{\mu \nu}+m_{V}^{2} e^{-2 \sigma(x)} V_{\nu} V^{\mu}\right) .
$$

The appearance of the factor $e^{-2 \sigma}$ in the mass term guarantees again the same autoparallel trajectories in the eikonal approximation as for spinless particles in the action (6).

Note that the scalar product factor $e^{-3 \sigma(x)}$ implies a coupling to torsion also for the massless vector bosons which is fully compatible with gauge invariance. Due to the symmetry between $Z$-boson and photon, this factor must be present also in the electromagnetic action.

5. Let us end by remarking that autoparallel trajectories may be considered as a manifestation of a nonholonomic mapping principle proposed in [3.2] which transforms classical equations of motion from flat space to spaces with curvature and torsion. This principle was an essential tool for finding the solution of a completely different fundamental problem, the path integral of the hydrogen atom [2].

Autoparallel trajectories are also the most natural trajectories if a space with torsion is constructed by a nonholonomic embedding a Riemann-Cartan space in a flat space [12]. The are, moreover, the only trajectories which do not violate the universality principle of spin and angular momentum in the coupling of fundamental particles to torsion. As was shown in [13], this principle greatly restricts such couplings, since the spin of a fundamental particle is always a fluctuating mixture of orbital and spin angular momenta of its constituents. This mixture cannot be resolved in a representation of the Poincaré group for the composite particle, since its states are labeled by the quantum numbers $s, s_{3}$ of the total spin. This blindness will be inherent in any gauge theory of this group, if it is to be compatible with the physics of elementary particles, as long as we do not possess an ultimate theory of these particles, which only string people claim to possess, in spite of a complete disagreement with spacetime dimensions and particle spectra of the world in which we live.

Acknowledgement:

The author is grateful to Prof. F.W. Hehl for numerous interesting discussions and to Dr. A. Pelster for useful comments.

[1] R. Utiyama, Phys. Rev. 101, 1597 (1956); T.W.B. Kibble, J. Math. Phys. 2, 212 (1961); F.W. Hehl, P. von der Heyde, G.D. Kerlick and J.M. Nester, Rev. Mod. Phys. 48, 393 (1976); F.W. Hehl, J.D. McCrea, E.W. Mielke and Y. Ne'eman, Phys. Rep. 258, 1 (1995).

[2] H. Kleinert, Path Integrals in Quantum Mechanics, Statistics and Polymer Physics, World Scientific, Singapore 1990 and 1995, Second extended edition, pp. 1-850 (http://www.physik.fu-berlin.de/ kleinert/kleiner_re.html\#b5).

[3] H. Kleinert, Nonholonomic Mapping Principle for Classical Mechanics in Spaces with Curvature and Torsion FU-Berlin preprint 1997 (APS E-Print aps1997sep03_002). Short version presented as a lecture at the Workshop on Gauge Theories of Gravitation, Jadwisin, Poland, 4-10 September 1997, Acta Phys. Pol. B 29, 1033 (1998) (gr-qc/9801003).

[4] F.W. Hehl, Phys. Lett. A 36, 225 (1971). See also Section VII in [3].

[5] P. Fiziev and H. Kleinert, Europhys. Lett. 35, 241 (1996) (hep-th/9503074). 
[6] H. Kleinert and A. Pelster, (gr-qc/9605028.

[7] Our notation of field theoretic and geometric quantities is the same as in the textbook H. Kleinert, Gauge Fields in Condensed Matter, Vol. II Stresses and Defects, World Scientific, Singapore 1989, pp. 744-1443 (http://www.physik.fuberlin.de/ ${ }^{\sim}$ kleinert/kleiner_re.html\#b2).

[8] See Section 11.4 in Ref. 2]. Note that the normalization of the $\sigma$-field is normalized differently from the present one by a factor $2 / 3$. There we introduced $\delta$ via the relation $S_{\mu \nu}{ }^{\nu}=\partial_{\mu} \sigma$, whereas here $S_{\mu \nu}{ }^{\nu}=(3 / 2) \partial_{\mu} \sigma$.

[9] H. Kleinert and A. Pelster, Novel Geometric Gauge-Invariance of Autoparallels, Lectures presented at Workshop Gauge Theories of Gravitation, Jadwisin, Poland, 4-10 September 1997, Acta Phys. Pol. B 29, 1015 (1998) (gr-qc/9801030).

[10] A. Saa, Mod. Phys. Lett. A8, 2565, (1993); ibid. 971, (1994); Class. Quant. Grav. 12, L85, (1995); J. Geom. and Phys. 15, 102, (1995); Gen. Rel. and Grav. 29, 205, (1997).

[11] P.P. Fiziev, Gen. Rel. Grav. 30, 1341 (1998) gr-qc/9712004), See also gr-qc/9808006

[12] H. Kleinert and S.V. Shabanov, gr-qc/9709067).

[13] H. Kleinert, Universality Principle for Orbital Angular Momentum and Spin in Gravity with Torsion gr-qc/9807021) 